INPLASY

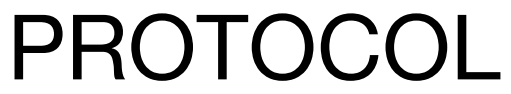

To cite: Xiong et al.

Effectiveness of Internet intervention for employees with depression: a systematic review and meta-analysis. Inplasy protocol 202160082. doi:

10.37766/inplasy2021.6.0082

Received: 22 June 2021

Published: 22 June 2021

Corresponding author: Juan Xiong

xiongjuan0@sina.com

Author Affiliation:

Chongqing University.

Support: None.

Review Stage at time of this submission: Data analysis.

Conflicts of interest:

None declared.

\section{Effectiveness of Internet intervention for employees with depression: a systematic review and meta-analysis}

Xiong, J1; Wen, J2; Pei, G3; He, D².

Review question / Objective: We aimed to systematically investigate the available evidence for Internet interventions in reducing depressive symptoms for employees.

Condition being studied: The effectiveness of Internet intervention for employees with depression remains unclear. Information sources: In this systematic review and metaanalysis, we searched MEDLINE (Ovid), EMBASE (Elsevier), PsycINFO (EbscoHost), the Cochrane Library (Wiley), and Web of Science for articles published in English from database inception to May, 2021.

INPLASY registration number: This protocol was registered with the International Platform of Registered Systematic Review and Meta-Analysis Protocols (INPLASY) on 22 June 2021 and was last updated on 22 June 2021 (registration number INPLASY202160082).

\section{INTRODUCTION}

Review question / Objective: We aimed to systematically investigate the available evidence for Internet interventions in reducing depressive symptoms for employees.

Condition being studied: The effectiveness of Internet intervention for employees with depression remains unclear.

\section{METHODS}

Search strategy: (Employees* OR staff* OR clerk $^{\star}$ ) and (Randomized Controlled Trial*

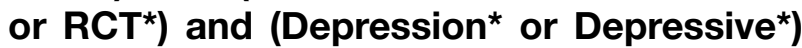
and (Digital intervention* or Computerized* or Computerized intervention* Internetdelivered* $^{*}$ or Internet intervention* or Smartphone intervention* or Game-based internet* ${ }^{\star}$ or Web-based intervention*).

Participant or population: The Employees. 
Intervention: The Internet interventions.

Comparator: Control group(eg, active control, treatment as usual, wait-list control).

Study designs to be included: We identified 21 eligible studies that were included in the meta-analysis. The included studies involved a total of 5898 participants (2813 who received Internet intervention and 3085 who were in the control group).

Eligibility criteria: 1) Outcome measures included the frequently used scales of measuring depression [eg, the Center for Epidemiological Studies Depression scale (CES-D), the Hospital Anxiety and Depression Scale (HADS), the Maslach Burnout Inventory-General Scale (MBI), Patient Health Questionnaire-9 (PHQ-9), the Beck Depression Inventory II (BDIII).et.al]; 2) Participants had to be employees, in order to maximise methodological quality participants in the original literature allocation had to be randomized controlled trial; 3) At least one control condition in the study had to be an inactive intervention (for example, waitlist, no intervention, placebo, monitoring, an attention control group) or another treatment (psychological or pharmacological); 4)The original article about employees needed to be available in English and published in a peer-reviewed journal and not be further analysis of data from a randomized controlled trial.

Information sources: In this systematic review and meta-analysis, we searched MEDLINE (Ovid), EMBASE (Elsevier), PsycINFO (EbscoHost), the Cochrane Library (Wiley), and Web of Science for articles published in English from database inception to May, 2021.

Main outcome(s): Depression

Additional outcome(s): The mental health problems.
Data management: The majority of interventions $(20 / 21,95 \%)$ were delivered via the Internet, and the most common intervention was internet-based cognitive behavioral therapy $(4 / 21,19 \%)$.

Quality assessment / Risk of bias analysis: Data were synthesized using a systematic narrative synthesis framework, and formal quality assessments were conducted to address the risk of bias.

Strategy of data synthesis: Heterogeneity between studies was substantial $\left(I^{2}=83 \%\right)$. There was no evidence of publication bias, and the quality of evidence according to the GRADE criteria was generally high.

Subgroup analysis: country (America; Australia; Germany; Japan; Netherlands; Sweden; UK); outcome measure (BDIII;CES-D;DASS HADS;OTHER;PHQ-9) control group (Active control; Treatment as usual; Waitlist control); Duration (<3 months; $\geq 3$ months).

Sensitivity analysis: To avoid the consequent potential for inflation of the treatment effect, we did a post-hoc sensitivity analysis by removing these studies from the meta-analysis.

Language: English.

Country(ies) involved: China.

Other relevant information: None.

Keywords: Depression; Employees; Internet intervention; Meta-analysis.

Contributions of each author:

Author 1 - Juan Xiong.

Email: xiongjuan0@sina.com

Author 2 - Jianlin Wen.

Email: Forrestwin2022@163.com

Author 3 - Guangshu Pei.

Email: pgs@cqu.edu.cn

Author 4 - Danqing He.

Email: hdqhoney@126.com 